\title{
Resveratrol as an Inhibitor of Pregnane X Receptor (PXR): Another Lesson in PXR Antagonism
}

\author{
Tomas Smutny ${ }^{1}$ and Petr Pavek ${ }^{1, *}$ \\ ${ }^{1}$ Centre of Drug-Dietary Supplements Interactions and Nutrigenetics, Faculty of Pharmacy, Charles University in Prague, \\ Heyrovskeho 1203, Hradec Kralove, Czech Republic, CZ500 05, Czech Republic
}

Received August 22, 2014

\begin{abstract}
The pregnane X receptor (PXR) plays a central role in the regulation of metabolism. Only few PXR antagonists have been described and the mechanism of PXR inhibition is mostly hypothetical or believed to be allosteric. Resveratrol is now being discussed as a novel agent that is capable of attenuating the PXR-inducible expression of the CYP3A4 gene, although the mechanistic explanation has not been determined. We discuss herein the effect of resveratrol on PXR in the context of our data from a ligand binding assay and with respect to the effect of resveratrol on various cellular signaling pathways that are known to affect PXR.
\end{abstract}

Keywords: nuclear receptor, Pregnane X Receptor (PXR), gene regulation

The pregnane $\mathrm{X}$ receptor (PXR, NR1I2) has been shown to be the primary regulator of major xenobioticmetabolizing enzymes, including CYP3A4, CYP2C9, CYP2B6, and UGT1A1. It is well established that the transcriptional activity of PXR is regulated by ligands via direct binding to the ligand binding domain (LBD). PXR binds a wide array of structurally distinct ligands into its large ligand-binding pocket (up to $1,150 \AA^{3}$ ). When a ligand is present, the AF-2 surface of the PXR LBD is stabilized in a conformation that allows the binding of transcriptional co-activators. However, in contrast to the numerous PXR agonists that have been discovered, only a few antagonists have been described and the mode of action of these antagonists remains unclear.

In 2001, ET-743 became the first PXR antagonist reported, subsequently followed by compounds such ketoconazole and its derivatives; coumestrol; sulforaphane and allyl isothiocyanate; metformin; the HIV protease inhibitor A792611; the naturally occurring lignan sesamin; camptothecin; algal caroteinoid fucoxanthin; silybin; and isosilibin (1). However, only a few PXR antagonists - coumestrol, sulforaphane, silybin, and isosilibin - have been studied employing a ligand

*Corresponding author. petr.pavek@faf.cuni.cz

Published online in J-STAGE

doi: 10.1254/jphs.14001LT binding assay with respect to their direct interaction with PXR LBD. Significantly, some of these compounds - ketoconazole, coumesterol, metformin - inhibit PXR transactivation via interference with $\mathrm{PXR}$ coactivators or through binding to the AF-2 domain of PXR independently of PXR LBD. It has been recently hypothesized that ketoconazole may affect PXR transcriptional regulation by binding to two potential and distinct "antagonistbinding pockets" outside the ligand binding pocket (2). Nevertheless, no PXR antagonist has been described to exert its effect within the bulky PXR binding pocket so far, which brings into question the ability of a smallmolecule antagonist to interact with PXR LBD.

Several studies have indicated that a number of cellular signaling pathways affected by small-molecule compounds modulate the function of PXR via a posttranslational phosphorylation-mediated mechanism with respect to both basal and ligand-activated transactivation. PXR has been found to be phosphorylated in three ways: by the protein kinase $\mathrm{A}$ (PKA) and the protein kinase $\mathrm{C}$ (PKC) signaling pathways, by cyclin-dependent kinases 2 (CDK2) and 5 (CDK5), and by p70 S6K, a downstream kinase of the PI3K-Akt pathway (3).

In the current issue of the Journal of Pharmacological Sciences, Deng et al. show evidence for the first time that trans-resveratrol (RES), a natural stilbenoid, attenuates the rifampicin-inducible expression of CYP3A4 through PXR (4). As a high concentration of RES is found in 
red wine, in red grapes, and in other fruits and since RES is present in numerous antioxidative and antiaging nutritional supplements, this finding may have significant clinical consequences in terms of drug-dietary supplement and food-drug interactions.

RES has been found to competitively inhibit cAMPdegrading phosphodiesterases, leading to elevated cAMP levels. The resulting activation of Epac1, a cAMP effector protein, increases intracellular $\mathrm{Ca}^{2+}$ levels, activates the $\operatorname{CamKK} \beta$-AMPK pathway as well as increases activity of SIRT1 deacetylase. In addition, numerous other cellular effects of RES have been reported in the literature, including controlling cellular calcium homeostasis, modulation of the transcription factors $\mathrm{NF}-\kappa \mathrm{B}, \mathrm{Nrf} 2$, and topoisomerase inhibition.

Therefore, a link can be hypothesized between the effects, on the one hand, of RES on cellular cAMP and $\mathrm{Ca}^{2+}$ levels or on the other hand, PKA and PKC signaling activation as the reason for the consequent suppression of PXR activity due to the phosphorylation of PXR via the signaling cascades. Obviously, further evidence is needed. RES may also indirectly influence PXR activity via NF- $\kappa$ B inhibition or via SIRT1 signaling since PXR interferes reciprocally with the cascades.

Using a Time Resolved-FRET based ligand binding cell-independent in vitro assay with recombinant human PXR, we observed a significant interaction of RES with the PXR ligand binding pocket within the concentration range of $0.1-20 \mu \mathrm{M}$. Consistent with previous reports by Kluth et al. (5), Jacobs et al. (6), Yu et al. (7), Dring et al. (8) as well as with our as yet unpublished data, both trans- and cis-RES to limited extent activate PXRinduced CYP3A4 transactivation and mRNA expression. All these data indicate the interaction of RES with PXR LBD. Further studies should consider RES as a partial agonist of PXR, both in activating PXR function, but also inhibiting the effect of other PXR agonists. Accepting this proposition can also explain discrepant data by Dring et al. (6), who did not observe the inhibition of the model high-affinity PXR agonist rifampicin at extremely high concentration $(25 \mu \mathrm{M})$ with RES.

Therefore, we can conclude based on current knowledge that RES may i) interact with the PXR LBD domain and/or ii) activate some protein kinase signaling cascades to suppress PXR activity in regulation of its target genes.
There is growing consensus that PXR inhibition may be an important mechanism in reversing cancer drug resistance and tumor growth, eliminating unwanted PXR-mediated drug-drug interactions, and alleviating PXR-induced hepatic steatosis. Thus new selective and non-toxic PXR antagonists are being investigated intensively. Primarily, however, there is urgent need for an increased understanding of the mechanisms of PXR antagonisms.

\section{Acknowledgments}

Supported by GACR303/12/0472 and SVV 170/50/33904-3 projects.

\section{Conflicts of Interest}

We declare no conflict of interest.

\section{References}

1 Mani S, Dou W, Redinbo MR. PXR antagonists and implication in drug metabolism. Drug Metab Rev. 2013;45:60-72.

2 Li H, Redinbo MR, Venkatesh M, Ekins S, Chaudhry A, Bloch $\mathrm{N}$, et al. Novel yeast-based strategy unveils antagonist binding regions on the nuclear xenobiotic receptor PXR. J Biol Chem. 2013;288:13655-13668.

3 Smutny T, Mani S, Pavek P. Post-translational and posttranscriptional modifications of pregnane $X$ receptor (PXR) in regulation of the cytochrome P450 superfamily. Curr Drug Metab. 2013;14:1059-1069.

4 Deng R, Xu C, Chen X, Chen P, Wang Y, Zhou X, et al. Resveratrol suppresses the inducible expression of CYP3A4 through the pregnane X receptor. J Pharmacol Sci. 2014;126:146-154.

5 Kluth D, Banning A, Paur I, Blomhoff R, Brigelius-Flohé R. Modulation of pregnane $\mathrm{X}$ receptor- and electrophile responsive element-mediated gene expression by dietary polyphenolic compounds. Free Radic Biol Med. 2007;42:315-325.

6 Jacobs MN, Nolan GT, Hood SR. Lignans, bacteriocides and organochlorine compounds activate the human pregnane $\mathrm{X}$ receptor (PXR). Toxicol Appl Pharmacol. 2005;209:123-133.

7 Yu C, Chai X, Yu L, Chen S, Zeng S. Identification of novel pregnane $\mathrm{X}$ receptor activators from traditional Chinese medicines. J Ethnopharmacol. 2011;36:137-143.

8 Dring AM, Anderson LE, Qamar S, Stoner MA. Rational quantitative structure-activity relationship (RQSAR) screen for PXR and CAR isoform-specific nuclear receptor ligands. Chem Biol Interact. 2010;188:512-525. 